\title{
Campus Second-hand Trading Market Application System
}

\author{
Li Zhu ${ }^{1, a}$, Lan Bai ${ }^{2, b}$
}

${ }^{1}$ College of Mathematics and Computer Science, Jiangxi Science and Technology Normal University, Nanchang, China

${ }^{2}$ Computer and information branch, Jiangxi Modern Polytechnic College, Nanchang, China a13184572225@163.com , b ilovexz0802@163.com

Keywords: Internet; Campus second-hand goods; Online trading; Struts framework; Application system

Abstract: The emergence of the Internet led to a period, also brought a lot of convenience to people's life, and the wave of Internet online shopping has gradually become another mainstream network service. School students now do not have a very good handling for second-hand resources, which not only wastes the resources, also can't save economic cost. Based on these characteristics, this paper studies and designs campus second-hand market application system, systematically expounds the implement and main functions of campus second-hand market application system. The paper designed and implemented commodity information browsing, commodity classification inquiries, online communication and background management system, and other functions. It provides a quick and convenient online trading platform mainly for students' second-hand goods, let the students spare yard realize its value, and let the students in need access to cheap and satisfied second-hand items. It is both environmental and economic, and has high practical value.

\section{INTRODUCTION}

As our country pushes forward the Internet, Internet industry flourishes, and online shopping has become an indispensable part of people's life. Most young people have the experience of shopping on the Internet. It is because there are so swollen and broad market prospects that make more and more entrepreneurship people can get through online platform. An environment-friendly society have been advocated, everyone should start from resource conservation. In school, especially many second-hand goods are thrown away in universities and colleges, which both damages the environment, and wastes of resources. For such rich second-hand resources, we can take reasonable way to handle it, build an Internet platform, make full use of the mature technology and social environment, let the students used resources can be reasonable shared and effectively used so as to make resources communication on campus, save cost, and make waste useful. So create school second-hand trading platform is feasible and has certain practical value.

\section{FEASIBILITY ANALYSIS}

A. Technical feasibility

Now many college students have laptops or desktop computers. From the aspects of software technology, programming language has been very mature, and the system function completely can be achieved. Use image creation tools and HTML, we can produce elegant and vivid or animated web pages independently.

B. Economic feasibility

The cost of this system lies in the upfront cost of software development, and system only requires students to have a computer to browse the web. The service object of the system is college students. For them, income is relatively small, second-hand goods have more markets for college students, which not only can save resources, but save money for low-income college students. If everyone shares resources, makes full use of this platform, the benefits will be much larger than the input, therefore, it is feasible in economy.

C. Operation feasibility

The system interface design fully takes into account the habits of users and administrators. Friendly interface is clear and beautiful, and also very simple for operation. Users only need a computer to browse the web to get into the system. 


\section{SYSTEM FUNCTION}

A. Campus second-hand trading market application system is a small e-commerce system based on JSP technology, which mainly provides quick online shopping environment similar to Taobao for college students, catering to the more and more popular e-commerce mode. College students use the system to realize registered users, browse through the second-hand goods released, query needed products' category and order information. You can also check your order and change your basic information. In addition, message boards and other humanized design let students enjoy shopping. In administrator module, there are administrator identification, management user, order, management of second-hand goods, and we can also manage message boards, maintain and update the system.

B. Security requirements include: users' browse, choose and buy goods, no right to modify and delete items; System administrator can manage the merchandise, member, and order information after log-in.

C. Users can buy goods after registration landing, and administrators will authenticate the identity of the user.

The whole system is mainly divided into two parts, users and administrators.

\section{FUNCTIONAL OPERATION PROCESS DESIGN}

First enter the log-in screen, enter the administrator user name and password, transmit data to the background, and match with data in the database. If the match is successful, jump to function interface; if the match is not successful, prompt incorrect password or user name, and return to the log-in screen enter again.

A. Add data process

Automatically generate number according to the classification, and then input data and system checks its legitimacy. If legal, record and write to the database; if not legal, prompt the user input is wrong, ask users to input again, and add data. The flow diagram is shown in Figure 1.

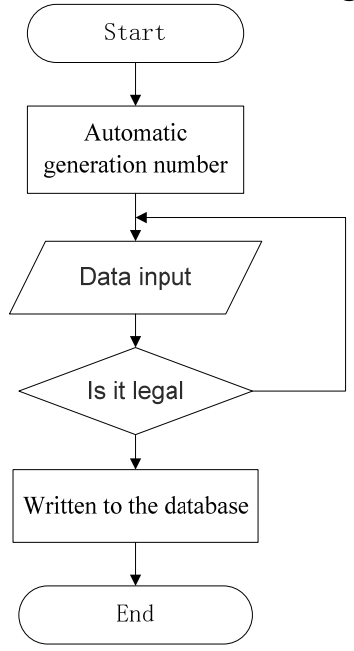

Figure 1 Add data flow chart

\section{B. Data modification process}

Select a record to modify and then input data. Check if the input data is legal. If legal, input data to database; if not legal, enter the new data again, until the data input is legal.

C. Delete data flow

Choose the record need to be deleted, then jump out of the options remind "delete records?" Select "no", do not delete, back to choose the record need to be deleted; select "yes", then update the database, delete the record need to be deleted.

\section{SYSTEM DESIGN IN DETAIL}

A. System e-r diagram

Administrators have a ID, user name, password, address, telephone and other attributes, which are 
generated automatically in the database ID, so they are not reflected in e-r diagram; Administrator ID is associated with released commodity ID, and commodity attribute released are: ID, name, model and introduction in detail; Each kind of commodity carries on the classification to facilitate management, and its serial number is associated with product ID.

Member information is relatively simple, whose main function is log-in, release information, shopping, business, and manage goods. Figure 2 is the system e-r diagram below.

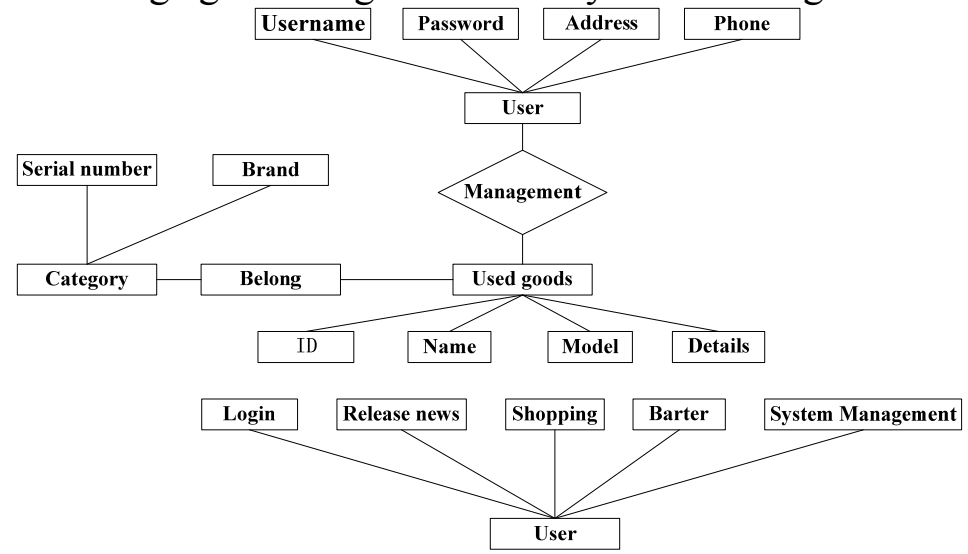

\section{B. Database design}

Figure 2 System e-r diagram

SQLserver is essentially a high performance, large capacity client server provided by Microsoft. DBMS relational database management system can support large throughput, transaction management which has been widely used as a background database. It can conceive and design database according to the overall design idea.

C. Database connection technology

Every page in the process of common web development uses the operation of database. This system uses data connection technology for JDBC connection technology. JDBC connection technology is a kind of technology which can provide all kinds of common database with seamless link. It not only can connect to database, and can perform corresponding SQL statements, and the model is shown in Figure 3.

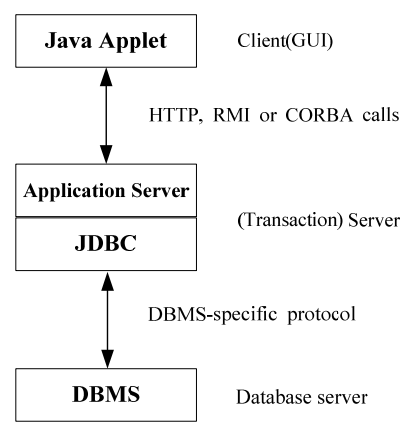

Figure 3 JDBC three layer models

The database connection is shown in Figure 4: 


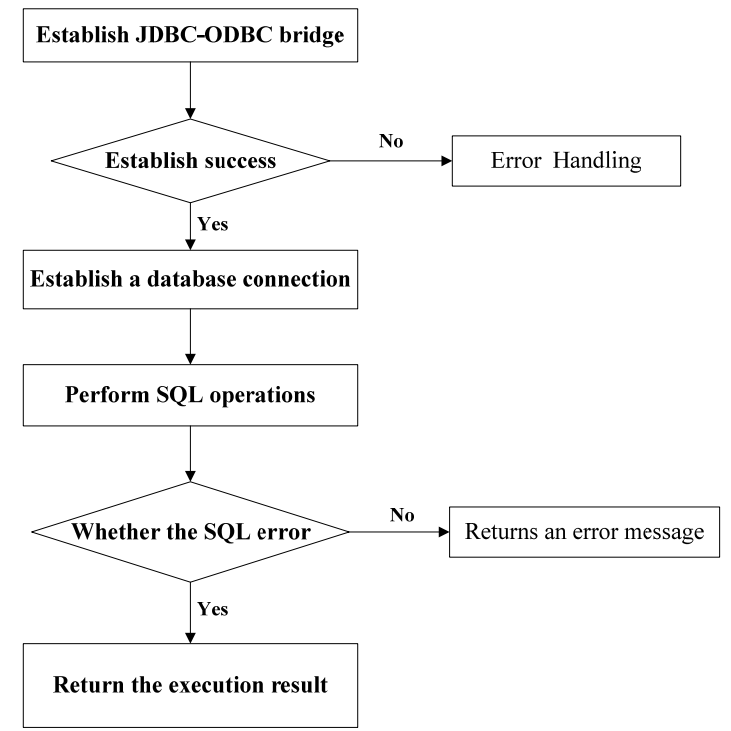

D. System interface code design

Figure 4 of the database connection handle

(1) User registration

New user registration must fill in the user name, password, etc. For college students, there are class, student number, etc., in log-in process; we also have the problem of password prompt. If lost password, we can retrieve password via password prompt problem.

(2) Second-hand goods release

To release second-hand goods, we need to fill in the details of the goods, including name, classification. The classification is based on the available options for the user to fill out in order to let buyers have more intuitive understanding of the commodity information, including second-hand goods' monographs, and price and so on. The last column is the introduction to let the seller add more detailed description of second-hand goods information, fill in all these information, then press "submit data", then the system will submit data records stored in the database, and buyers can find the released goods in the classification of goods.

(3) System management module design

After entering the existing account $\log$ in, users need to fill in user name and password, and still need to fill out verification code. If the password is lost, press "retrieve password" option to retrieve password by answering questions.

System home page has three sections: commodity classification, site, and the latest released goods. The page is concise and clear, and the top is the entrance of several versions, including home page, news and information, commodity information, commodity classification, information query, exchange goods, administrator log-in member center, as well as the background.

After administrator logged in, he can enter the background management page. Function module is on the left, and the details of the operation are on the right. Administrator can add and delete users, and modify their own information and announcement. Announcement can be released in the main page to see some good goods. It is convenient for user to understand the latest release information; in news and information module, the administrator can release some current news or content people are interested in; in commodity information management module, the administrator can release the information for review and classify, so that users can search the goods they need faster. In registered member management, the administrator block or remove a member who release bad information, add or remove the classification of goods.

System management interface core code:

$<\% @$ page language="java" import="java.util.*,com.util.*"

contentType="text $/ \mathrm{html} ;$ charset $=\mathrm{gb} 2312 " \%>$

$<$ jsp:useBean id="sn" scope="page" class="com.bean. SystemBean" /

$<\%$

List list $=$ sn.getSiteInfo(); 
String $\operatorname{str}=$ list.get $(0) \cdot$ toString () ;

String path $=$ request.getContextPath () ;

String

basePath

request.getScheme()+"://"+request.getServerName()+":"+request.getServerPort()+path+"/";

$\%>$

$<$ !DOCTYPE HTML PUBLIC "-//W3C//DTD HTML 4.0 Frameset//EN">

$<$ HTML $><$ HEAD $><$ TITLE $>$ website backstage management $--.--<\%=$ STR $\%></$ TITLE $>$

The $<$ META HTTP - equiv $=$ the content-type Content $=$ "text/HTML; charset $=$ gb2312" $>$

The $<$ META content $=$ "MSHTML 6.00.2900.3243" name $=$ the GENERATOR $></$ HEAD $>$

The $<\%$

String username $=($ String $)$ session. The getAttribute (" user ");

If (username $==$ null) \{

Response. SendRedirect (path + "/ error. JSP");

\}

The else \{

String dir $=$ sn. GetDir ();

$\%>$

$<$ FRAMESET border $=0$ frameSpacing $=0$ rows $=65, *, 33$ frameBorder $=0>$

$<$ FRAME name $=$ FrameTop SRC $="<\%=$ basePath $\%><\%=\%$ dir $>/$ iframe/top. JSP" frameBorder $=$ no noResize scrolling $=$ no $>$

$<$ FRAMESET border $=0$ name $=$ FrameMain frameSpacing $=0$ frameBorder $=0$ cols $=165, *>$

$<$ FRAME name $=$ LeftFrame marginWidth $=0$ marginHeight $=0 \mathrm{SRC}="<\%=$ basePath $\%><\%$

$=\%$ dir $>/$ iframe left. The JSP" frameBorder $=$ no scrolling $=$ no $>$

$<$ FRAMESET frameSpacing border $=0=0$ rows $=*, 26$ frameBorder $=0>$

$<$ FRAME name $=$ MainFrame marginWidth $=0$ marginHeight $=0 \mathrm{SRC}="<\%=$ basePath $\%>$

$<\%=\% \operatorname{dir}>/$ iframe $/$ main JSP" frameBorder $=$ no noResize scrolling $=$ yes $>$

$<$ FRAME name $=$ BottomFrame marginWidth $=0$ marginHeight $=0$ SRC $="<\%=$ basePath $\%>$ $<\%=\%$ dir $>/$ iframe/help. JSP" frameBorder $=$ no scrolling $=$ no $>$

$</$ FRAMESET $>$

$</$ FRAMESET $>$

$<$ FRAME name $=$ FrameBottom marginWidth $=0$ marginHeight $=0$ SRC $="<\%=$ basePath $\%>$ $<\%=\%$ dir $>/$ iframe/foot. The JSP" frameBorder $=$ no noResize scrolling $=$ no $>$

$</$ FRAMESET $>$

The $<\%\} \%>$

The $</$ HTML $>$

\section{E. System test}

In system program code writing process, there are often all sorts of mistakes, which needs program debugging in the code complete process of each part. Some mistakes can use debugging tools. According to the prompt, find out the cause of the error, make error code correction and run again, until the program can normal boot and achieve the expected function and then get in next function code writing. Considering the safety, the decryption process is more complex, which need to consume a little running time; In the process of encoding, set aside enough interface to deal with the module needed to be added for normal maintenance and operation of the whole system in the future.

\section{ConCLuSion}

The development of the Internet greatly changes people's life, and brings a lot of convenience. This system is to develop a second-hand market application system for students, which uses the Internet characteristics. It provides a quick and convenient online trading platform for current students in school, which can let the students spare yard to realize its value, let the students in need access to cheap and satisfied second-hand items. Give full play to its performance, save the costs, and make 
reasonable use of network resources.

\section{REFERENCE}

[1] Wang Jing. College students' online shopping consumption: The status quo, problems and countermeasures [J]. Journal of Economic Study Tribune. 2015.1 (1) : 82-83

[2] Xie Panpan. Campus second-hand trading market analysis [J]. Journal of Jiangsu Business Theory, 2009, (9) : 269-269.

[3] An Yinglian Yao Ruixia, Tian Rongpai, etc. Teaching design and practice of "web page making" [J]. Journal of Computer Education, 2005, (8) : 29 and 30.

[4] Zhang Nanping, Zhu Fuli. The research and application of Struts framework based on MVC pattern [J]. Computer Technology and Development, 2006, 16 (3) : 229-231.

[5] Chen Yiming. SQL Server database application skill study [J]. Science, Technology and Engineering, 2008, 8 (12) : 3334-3337.

[6] Liu Chuan. JDBC technology application in electronic commerce [J]. Journal of Small and Medium-sized Enterprise Management and Technology, 2010, (36) : 288-288.

[7] Long Jun. The working principle and implementation of JDBC technology [J]. Science and Technology Square. 2004 (7) : 16 to 18.

[8] Zhao Junfeng. Java Web application development case tutorial: JSP + Servlet + JDBC and AJAX based on the MVC pattern [j]. Tsinghua University Press, 2012 (1) : 68-70

[9] Zhou Yu, Zhou Guoqing, Xi Wenjun etc. Summary of software testing technology [J]. China Measurement Technology, 2005, 31 (3) : 56-58.

[10] Yang Genxing, Zong Yuwei. Software testing uncertainty research and solutions [J]. Computer Engineering Calculation,. 2004, 30 (7) : 52-54. 